\title{
Bone Activity Biomarkers and Bone Mineral Density in Children with Chronic Kidney Disease
}

\author{
Yasser F Ali ${ }^{\mathrm{a}}$, Amal M Abdel-Latif ${ }^{\mathrm{a}}$, Mohamed A El-Koumi ${ }^{\mathrm{a}}$, Adel AM Ghorab ${ }^{\mathrm{b} *}$, Hany A labib ${ }^{\mathrm{c}}$ \\ Departments of (a) Pediatrics, (b) Internal Medicine and (b) Clinical Pathology, Faculty of Medicine, Zagazig University, Egypt
}

\begin{abstract}
Introduction: Chronic kidney disease mineral and bone disorder (CKD-MBD) is a spectrum of bone minerals changes that range from the high turnover lesions of secondary hyperparathyroidism to the low turnover lesions of adynamic bone disease. Bone biopsy is the gold standard for the diagnosis, but it is not routinely performed because it is an invasive technique.
\end{abstract}

Methods: Fifteen CKD children on regular hemodialysis (group I) and fifteen CKD children on conservative management (group II) were selected from the nephrology clinics of Zagazig University Hospitals along with fifteen age and sex-matched healthy controls. Participants were subjected to biochemical assessment that included osteocalcin (OC), total and bone-specific alkaline phosphatase (tALP and bALP), isomerized beta form of type I collagen cross-linked telopeptide ( $\beta$-Crosslaps) and intact parathyroid hormone (iPTH) levels. Patients with CKD also had their bone mineral density (BMD) measured using dual energy X-ray absorptiometry (DEXA) at lumbar spine and femoral neck.

Results: Serum $\beta$-Crosslaps, OC and bALP were significantly higher in patient groups than controls and in group I compared to group II . There was a negative significant correlation between mean Z-score at lumbar spine and bALP, OC and iPTH in group I and with $\beta$-Crosslaps in both patient groups. The mean Z-score at femoral neck correlated negatively with bALP in group $\mathrm{I}$, with OC in group II and with iPTH and $\beta$-Crosslaps in both groups.

Conclusion: Biochemical bone markers and assessment of BMD in patients with CKD may have a role in the early detection of CDK-MBD.

\footnotetext{
* Corresponding author; Department of Medicine, Faculty of Medicine, Zagazig University, Egypt.

E mail: dodo_senior@yahoo.com
}

Keywords: Bone Biomarkers; Bone Mineral Density; Chronic Kidney Disease; Mineral and Bone Disorder

\section{The authors declared no conflict of interest}

\section{Introduction}

Disorders of bone and mineral metabolism in patients with chronic kidney disease (CKD) represent a spectrum of skeletal disorders ranging from high-turnover lesions of secondary hyperparathyroidism to low-turnover lesions of osteomalaica and adynamic bone disease. The bone lesions are observed in patients with CKD from early stages (CKD stage 2) and are associated with significant morbidity $[1,2]$.

Disturbances in mineral and bone metabolism have traditionally been termed renal osteodystrophy (ROD) and classified based on bone biopsy findings. It is recommended that the term ROD be used exclusively to define alterations in bone morphology associated with $\mathrm{CKD}$, which can be further assessed by histomorphometry. The term CKD-Mineral and Bone Disorder (CKD-MBD) is used to describe a broader clinical syndrome that develops as a systemic disorder of mineral and bone metabolism due to CKD, which is manifested by abnormalities in bone and mineral metabolism and/or extra-skeletal calcification [3].

The remodeling cycle in normal bone lasts from 4 to 8 months and includes several phases: (1) an activation phase of osteoclasts; (2) a resorption phase in which osteoclasts erode the bone surface; (3) a reversal phase in which resorption end and osteobasts are recruited; (4) unmineralized matrix (Osteoid) synthesized by osteoblasts; and (5) mineralization of the osteoid tissue. Each of these steps (or several of them) are frequently compromised in uremic patients [4].

Bone biopsy is the gold standard for diagnosing ROD, but it is rarely used because it is an invasive procedure that needs special equipment. Consequently, in recent years, 
Table 1: Demographic and laboratory characteristics of the studied groups

\begin{tabular}{llll}
\hline Characteristic(s) & Group I (n=15) & Group II (n=15) & Controls $(\mathbf{n = 1 5})$ \\
\hline Age (years), mean \pm SD & $13.2 \pm 2.56$ & $12.1 \pm 3.2$ & $12.0 \pm 3.2$ \\
Male gender, number (\%) & $5(33.3 \%)$ & $6(40 \%)$ & $7(46.7 \%)$ \\
Onset of CKD (months), mean \pm SD & $25.3 \pm 11.5$ & $13.6 \pm 3.1$ & - \\
BUN (mg/dl), mean \pm SD & $67.7 \pm 11.3$ & $61 \pm 16.5$ & $10 \pm 2.9^{*}$ \\
Creatinine (mg/dl), mean \pm SD & $7.7 \pm 1.6$ & $2.7 \pm 1.4^{\dagger}$ & $0.96 \pm 0.3^{*}$ \\
Calcium (mg/dl), mean \pm SD & $7.8 \pm 1.1$ & $7.7 \pm 1.2$ & $9.6 \pm 0.6^{*}$ \\
Phosphorus (mg/dl), mean \pm SD & $6.2 \pm 1.5$ & $6.6 \pm 1.8$ & $4.6 \pm 0.7^{*}$ \\
iPTH (pg/ml), mean \pm SD & $323 \pm 156$ & $218.5 \pm 110.4^{\dagger}$ & $35.4 \pm 12.1^{*}$ \\
\hline
\end{tabular}

BUN: blood urea nitrogen; iPTH: intact parathyroid hormone

$* \mathrm{P}<0.001$ when control group was compared with group I or group II

$\dagger \mathrm{P}<0.05$ when group I was compared with group II

some biochemical markers of bone turnover have been used and tested in the evaluation of bone remodeling in uremic patients $[5,6]$.

The ideal biochemical bone marker should be unique to bone, reflect total skeletal activity and well correlated with histomorphometric and radiocalcium kinetic results [7].

Several enzymes and matrix proteins synthesized by osteoblasts, and some protein fragments released after bone matrix breakdown during the resorption process have been proposed as serum biochemical markers of bone formation. These include: bone-specific alkaline phosphatase (bALP), total alkaline phosphatase (tALP), osteocalcin (OC) and procollagen type I carboxyterminal propeptide (CTx). Moreover, bone resorption biomarkers [tartrate-resistant acid phosphatase (TRAP), isomerized beta form of type I collagen cross-linked telopeptide ( $\beta$-Crosslaps), and $\beta 2$-microglobulin] as well as cytokines and growth factors are involved in bone remodeling [8-10].

Serum total ALP and its bone specific isoenzymes are frequently used to monitor bone formation in patients with kidney disease since ALP is one of the few bone markers that is not influenced by variations in kidney function $[11,12]$.

Osteocalcin (OC) is a non-collagenous calcium-binding bone protein found in bone. It is secreted by osteoblasts and its molecular weight is 5800 daltons. It accounts for approximately $1 \%$ of total proteins in human. Osteocalcin may function as a negative regulator of bone formation, although its exact role is unknown [13]. OC is released into the circulation from the bone matrix during bone resorption and hence is considered a marker of bone turnover, rather than a specific marker of bone formation [14].

Type I collagen is the main collagen type found in mineralized bone. Especially relevant collagen type I fragments are the C-terminal telopeptides (CTx). In the C-terminal telopeptides the alpha aspartic acid converts to the beta form of aspartic acid as the bone ages. These isomerized telopeptides ( $\beta$-crosslaps) are specific for the degradation of type I collagen dominant in bone. Elevated serum concentration of $\beta$-crosslaps have been reported in patients with increased bone resorption and its serum level return to normal during resorption inhibition therapy [15].

Secondary hyperparathyroidism describes a complex alteration in bone and minerals metabolism that occurs as a direct result of CKD. Recently, assays have been developed for determination of intact parathyroid hormone (iPTH), that is the biologically active molecule which gives better possibilities to correlate with bone histomorphometry [16]. In vivo, increased PTH levels stimulate bone resorption by increasing the osteoclast cell number and activity. PTH also inhibits osteoprotegerin (OPG), a cytokine which can inhibit the production of osteoclasts, leading to a net catabolic effect on bone [17].

BMD can be measured using dual energy X-ray absorptiometry (DEXA) which is quick, non-invasive, accurate and associated with minimal exposure to radiation. BMD assessment in children with $\mathrm{CKD}$ is important because bone mass later in life is dependent upon the peak bone mass achieved in adolescence, and because low BMD is associated with increased morbidity 
Table 2: Etiology of chronic kidney disease (CKD) in patient groups

\begin{tabular}{lll}
\hline Etiology of CKD & Group I & Group II \\
\hline Congenital nephrosis & 0.0 & 6.7 \\
Glomerulonephritis & 13.3 & 13.3 \\
Hemolytic uremic syndrome (HUS) & 0.0 & 13.3 \\
Reflux nephropathy & 20.0 & 6.7 \\
Urinary tract infections & 6.7 & 0.0 \\
Systemic lupus erythematosus (SLE) & 6.7 & 13.3 \\
Steroid resistant nephrotic syndrome & 6.7 & 13.3 \\
Unknown etiology & 46.6 & 33.3 \\
\hline
\end{tabular}

$\mathrm{P}=0.53$

and mortality, although this has only been demonstrated in adults $[18,19]$.

This work aimed to study the changes in the levels of some biochemical markers of bone turnover and formation as well as their correlation with BMD, in both dialytic and predialytic children with CKD.

\section{Methods}

This cross sectional study was carried out in the nephrology units and out-patient clinics of pediatric and internal medicine departments, Zagazig University Hospital, during the year 2009. Thirty patients (11 males and 19 females) of ages ranging from 6 to 16 years were enrolled in the study. Ethical approval was obtained from the local research ethics committee and parents of all children gave informed written consent prior to the study. Glomerular filtration rate (GFR) was determined by ${ }^{99 \mathrm{~m}} \mathrm{Tc}-$ labelled diethylenetriamine penta-acetate $\left.{ }^{99 \mathrm{~m} T c-D T P A}\right)$ [20]. The patients were divided into two groups:

Group I: included 15 patients (5 males and 10 females) of mean age $13.2 \pm 2.56$ years with end stage renal disease (GFR: $<15 \mathrm{ml} / \mathrm{min} / 1.73 \mathrm{~m}^{2}$ ) on regular hemodialysis.

Group II: included 15 patients ( 6 males and 9 females) of mean age $12.1 \pm 3.2$ years in predialytic stage (GFR: $15-75 \mathrm{ml} / \mathrm{min} / 1.73 \mathrm{~m}^{2}$ ) on conservative management.

Fifteen healthy children ( 7 males and 8 females) of mean age $12.0 \pm 3.2$ years were recruited as controls.

\section{Exclusion criteria}

- Patients who were taking medication known to influence bone metabolism (corticosteroids, anticoagulants) within the last year.
- Patients who were on protein restricted diet.

- Patients who underwent parathyroidectomy.

- Patients with biochemical evidence of liver disease.

- Patients with malignant diseases.

- Patients receiving aluminum-containing phosphate binders.

All the study populations were subjected to full clinical history taking and physical examination as well as biochemical assessment including: serum calcium and phosphorus levels, serum creatinine and BUN, osteoblastic activity (by measuring serum OC, tALP and bALP), osteoclastic activity (by measuring $\beta$-Crosslaps), and serum iPTH.

Venous blood samples were withdrawn and collected in calcium free tubes and left at $37^{\circ} \mathrm{C}$ until clot retraction. Clear sera were separated after centrifugation at 2000 $\mathrm{rpm}$ for $15 \mathrm{~min}$, divided and stored at $-20^{\circ} \mathrm{C}$ till time of analysis. Fresh serum samples were used for assay of calcium, phosphorus, creatinine and BUN. Analysis methods included the following:

1. Serum calcium, phosphorus, creatinine and BUN were determined using ADVIA 1650 Autoanalyzer (Siemens Medical Solutions Diagnostic, USA).

2. Determination of tALP and bALP was based on the fact that the bone isoenzymes of ALP are heat labile and cannot withstand heating at $56^{\circ} \mathrm{C}$ for 10 minutes so that residual activity after heating for 10 minutes represents the other isoenzymes. tALP activity was measured first then heat for 10 minutes at $56^{\circ} \mathrm{C}$ was applied. The residual activity was measured and subtracted from the total activity to obtain the bALP activity. The percentage of bone isoenzyme activity was calculated. The normal bone ALP isoenzymes activity in children and adolescents is $85 \%$ of tALP [21].

3. Osteocalcin and $\beta$-crosslaps were assayed by electrochemiluminescence immunoassay (Roche Diagnostics Co., Gmbl, D-68298 Manheim) for use on Elecsys 2010 immunoassay analyzer.

4. Concentrations of iPTH were determined by automated chemiluminescence immunoassay on Immulite analyzer (Diagnostic products corporation, Los Angles, CA, USA) [22] .

Assessment of bone mineral density (BMD), using dual energy X-ray absorptiometry (DEXA, Lunar DPX-1Q system, USA), was performed only for patient groups. BMD of the lumbar spine (L2-L4) and femoral neck was determined in the supine position. BMD results were expressed as age and gender specific Z-scores. 
Table 3. Biochemical bone markers and BMD in studied groups

\begin{tabular}{llll}
\hline Variable(s) & Group I $(\mathbf{n}=\mathbf{1 5})$ & Group II $(\mathbf{n}=\mathbf{1 5})$ & Control $(\mathbf{n}=\mathbf{1 5})$ \\
\hline OC (ng/ml), mean \pm SD & $247.6 \pm 61.9$ & $171.8 \pm 23.7^{*}$ & $55.1 \pm 18.5 \dagger$ \\
$\beta$-Crosslaps (ng/ml), mean \pm SD & $4.3 \pm 1.4$ & $1.8 \pm 0.6^{*}$ & $0.8 \pm 0.3 \dagger$ \\
tALP (IU/L), mean \pm SD & $1331 \pm 425.4$ & $1019 \pm 729.2$ & $232.3 \pm 40.5 \dagger$ \\
bALP (IU/L), mean \pm SD & $788.3 \pm 170.9$ & $524 \pm 257^{*}$ & $209.6 \pm 53.4 \dagger$ \\
BMD (hip) Z-score, mean \pm SD & $-3.17 \pm 1.1$ & $-2.54 \pm 0.9^{*}$ & \\
BMD (lumbar) Z-score, mean \pm SD & $-2.74 \pm 0.9$ & $-1.90 \pm 0.8^{*}$ & \\
\hline
\end{tabular}

OC: Osteocalcin; $\beta$-Crosslaps: Isomerized beta form of type I collagen cross-linked telopeptide; tALP: Total alkaline phosphatase; bALP: Bone specific alkaline phosphatase; BMD: Bone mineral density

$* \mathrm{P}<0.05$ when group I was compared with group II

$\dagger \mathrm{P}<0.001$ when control group was compared with group I or group II

Table 4. Correlation coefficient between $\mathrm{Z}$-score of bone mineral density and bone biomarkers in the studied patients

\begin{tabular}{lllll}
\hline Biochemical markers & \multicolumn{2}{l}{$\begin{array}{l}\text { Correlation coefficient with Z-score } \\
\text { at lumbar spine }\end{array}$} & $\begin{array}{l}\text { Correlation coefficient with Z-score } \\
\text { at femoral neck }\end{array}$ \\
\cline { 2 - 5 } & Group I & Group II & Group I & Group II \\
\hline OC & $-0.48^{*}$ & -0.22 & -0.1 & $-0.46^{*}$ \\
$\beta$-Crosslaps & $-0.69 \dagger$ & $-0.52^{*}$ & $-0.61 \dagger$ & $-0.49^{*}$ \\
tALP & -0.19 & -0.39 & -0.32 & -0.21 \\
bALP & $-0.46^{*}$ & -0.33 & $-0.43^{*}$ & -0.15 \\
iPTH & $-0.53^{*}$ & -0.2 & $-0.67 \dagger$ & -0.32 \\
\hline
\end{tabular}

OC: Osteocalcin; $\beta$-Crosslaps: Isomerized beta form of type I collagen cross-linked telopeptide; tALP: Total alkaline phosphatase; bALP: Bone specific alkaline phosphatase

* Statistically significant, $\mathrm{P}<0.05$

$\dagger$ Statistically significant, $\mathrm{P}<0.001$

Z-scores were calculated from the following equation:

Z-score $=\left[\mathrm{BMD}\left(\mathrm{g} / \mathrm{cm}^{2}\right)\right.$ of patient $-\mathrm{BMD}$ predicted for age and sex]/ SD for BMD for age and sex matched.

$\mathrm{Z}$-scores equal to or greater than -1 were considered normal, $\mathrm{Z}$-scores lower than -1 and equal to or greater than -2 were considered to indicate osteopenia, and z-scores less than -2 were considered to indicate severe osteopenia [23].

Data were analyzed using Statistical Package for the Social Science (SPSS) version 14 for windows. Values were expressed as percentage or mean \pm standard deviation $(\mathrm{X} \pm \mathrm{SD})$. All statistical comparisons were performed using the student's t-test, chi-square and ANOVA. Linear correlations and regression were used to test the correlations between Z-score and other biochemical markers. P-values less than 0.05 were considered statistically significant.

\section{Results}

Table 1 shows the demographic and biochemical profiles of studied groups. There was no difference in mean age or gender distribution between the three groups. Groups I and II had similar serum calcium and phosphorus levels, but group I had significantly higher iPTH level.

Table 2 demonstrates the causes of renal failure in groups I and II. Unknown etiology for CKD represents the highest percentage in both $(46.6 \%$ and $33.3 \%$, respectively) and the distribution of underlying etiology was not significantly different between the two groups.

The biochemical markers of bone turnover and formation (OC, $\beta$-Crosslaps, tALP and bALP) were significantly higher in patient groups compared to controls $(\mathrm{p}<0.001)$. Also, significant differences between group I and group II as regard OC, $\beta$-Crosslaps and bALP were observed ( $p$ $<0.05$ ). The mean Z-score at lumbar spine and femoral 
neck was significantly different between patient groups (Table 3).

Z-score at lumbar spine was significantly and negatively correlated with OC, bALP and iPTH in group I and with $\beta$-Crosslaps in both groups. Z score at femoral neck was significantly and negatively correlated with bALP in group I, with OC in group II and with $\beta$-Crosslaps and iPTH in both groups (Table 4).

\section{Discussion}

Abnormalities in mineral metabolism and bone structure are almost universal findings with progressive CKD [24]. The term ROD is to be used exclusively to define alterations in bone morphology associated with CKD. The changes that occur in the homeostatic mechanisms that regulate serum concentrations of calcium and phosphate lead to the development of ROD [25]. Clinical symptoms of ROD are usually not present until advanced ROD is manifested so, early and accurate assessment of skeletal system in uremic patients is essential [26].

In this study BUN, serum creatinine, phosphate and $\mathrm{iPTH}$ were significantly higher while serum calcium levels were significantly lower in patient groups than controls. This is similar to what has been reported in many studies [27, 28], documenting iPTH hypersecretion and abnormal phosphate and calcium levels in CKD.

In our present study, on comparing dialytic patients (group I) and predialytic patients (group II), there were no significant differences as regard etiology of CKD. However, unknown etiology for CKD remained the commonest cause in both groups $(46.7 \%$ and $33.3 \%$ in groups I and II, respectively). Ahmadzadeh et al [29] reported in a study performed in South Western Iranian children that the commonest causes of CKD were reflux nephropathy, hypo/dysplastic kidney, hereditary nephropathy and obstructive uropathy.

The high frequency of CKD-BMD and ROD in children with CRF is likely related to the high rate of bone growth and the remodeling processes that are characteristic of the immature skeleton, which render it more sensitive to calcium/phosphate disturbance [30].

In our study, both biochemical markers of bone formation (OC, tALP, bALP) and bone resorption ( $\beta$-Crosslaps) were significantly higher in patient groups than controls, which may reflect higher rates of bone formation and degradation in patient groups. Meanwhile, bALP, OC and $\beta$-Crosslaps were significantly higher in patients with end stage renal disease (ESRD) than those in predialytic stage. Similar results were reported by other studies [7,31]. Magnusson et al [32] suggested that elevation of OC, and ICTP in patients dialyzed by both hemodialysis and peritoneal dialysis, may be partially due to the fact that these markers are cleared by the kidneys. The increase in bone turnover in these patients is due to the disturbance involving phosphate excretion, vitamin D3 metabolism, hypocalcemia, increased PTH and acid base disturbance which are known patho-physiological factors leading to destruction of bone microarchitecture and subsequently increase bone turnover [33].

DEXA was performed for our patient groups at both femoral neck and lumbar spine. The mean Z-score was significantly higher in group I than in group II. Similar results were also obtained by other studies [34, 35].

A study performed in Mansoura Children University Hospital that enrolled 21 children with predialysis CKD and 44 children with ESRD documented osteopenia in $13(62 \%)$ predialysis patients and $26(59.1 \%)$ patients with ESRD, with no significant correlation between $\mathrm{Z}$-score and the duration of CKD or estimated creatinine clearance [23].

In this study we found a significant negative correlation between mean Z-score at lumbar spine and bALP, OC and iPTH in group I and with $\beta$-Crosslaps in both patient groups. The mean $\mathrm{Z}$-score at femoral neck showed a statistically negative significant correlation with bALP in group I, with OC in group II and with iPTH and $\beta$-Crosslaps in both groups.

Bakr [23] reported negative correlations between Z-score and $\mathrm{iPTH}$, phosphate, bALP, and positive correlation with calcium, but no significant correlation with OC. Also, Urena et al [36] reported that BMD and Z-score were inversely correlated with bALP while Waller et al [22] found a significant correlation only with iPTH. Boot et al [37] concluded that the mean BMD of children with CRF did not differ from healthy controls. This discrepancy may be related to the small number of patients in each of these studies, varying patient characteristics, differences in therapeutic management and variability in bone mineralization and skeletal maturity due to differences in sexual maturation.

\section{Conclusion}

The studied biochemical bone markers (OC, $\beta$-Crosslaps, tALP and bALP) were increased in patients with CKD, both in dialytic and pre-dialytic stages. The absolute mean value of Z-score was significantly higher in dialytic than pre-dialytic patients with CKD and it showed a significant negative correlation with most of biochemical bone markers.

CKD-MBD starts early in the course of CKD, long before symptoms occur. It leads to bone deformities, fractures, bone pain and growth failure. Early and regular 
assessment of biochemical bone markers and BMD in patients with CKD should be considered to prevent the irreversible disabilities that may result from delayed treatment.

\section{References}

1. Moe SM, Drüeke T, Lameire N, Eknoyan G. Chronic kidney disease-mineral-bone disorder: A new paradigm. Adv Chronic Kidney Dis. 2007;14:3-12.

2. Kovesdy CP, Kalantar-Zadeh K. Bone and mineral disorders in pre-dialysis CKD. Int Urol Nephrol. 2008; 40:427-40.

3. Moe S, Drüeke T, Cunningham J, Goodman W, Martin K, Olgaard K, Ott S, Sprague S, Lameire N, Eknoyan G; Kidney Disease: Improving Global Outcomes (KDIGO). Definition, evaluation, and classification of renal osteodystrophy: A position statement from Kidney Disease: Improving Global Outcomes (KDIGO). Kidney Int. 2006 Jun;69(11):1945-53.

4. Eknoyan G, Levin A, Levin N. Bone metabolism and disease in chronic kidney disease. Am J Kidney Dis. 2003;42:1-201.

5. MartinKJ, OlgaardK, CoburnJW, Coen GM, Fukagawa M, Langman C, Malluche HH, McCarthy JT, Massry SG, Mehls O, Salusky IB, Silver JM, Smogorzewski MT, Slatopolsky EM, McCann L; Bone Turnover Work Group. Diagnosis, assessment, and treatment of bone turnover abnormalities in renal osteodystrophy. Am J Kidney Dis. 2004 Mar;43(3):558-65.

6. Ho LT, Sprague SM. Percutaneous bone biopsy in the diagnosis of renal osteodystrophy. Semin Nephrol 2002; 22(3): 268-75.

7. Ferreira A. Serum markers of bone turnover in the diagnosis of renal osteodystrophy. Rev Port Nefrol Hipert. 2005;19(1):57.

8. Kleerekoper M. Biochemical markers of bone turnover: why theory, research, and clinical practice are still in conflict. Clinical Chemistry. 2001; 47:1347-49.

9. Seibel MJ. Clinical use of markers of bone turnover in metastatic bone disease. Nat Clin Pract Oncol. 2005;2:504-17.

10. ChailurkitLO, SuthutvoravutU, Mahachoklertwattana P, Charoenkiatkul S, Rajatanavin R. Biochemical markers of bone formation in Thai children and adolescents. Endocr Res. 2005;31(3):159-69.

11. Blayney MJ, Pisoni RL, Bragg-Gresham JL, Bommer J, Piera L, Saito A, Akiba T, Keen ML, Young EW, Port FK. High alkaline phosphatase levels in hemodialysis patients are associated with higher risk of hospitalization and death. Kidney Int. 2008 Sep;74(5):655-63.

12. Haarhaus M, Fernström A, Magnusson M, Magnusson P. Clinical significance of bone alkaline phosphatase isoforms, including the novel $\mathrm{B} 1 \mathrm{x}$ isoform, in mild to moderate chronic kidney disease. Nephrology Dialysis Transplantation. 2009;24(11):3382-89.

13. Garnero P, Grimaux M, Seguin P, Delmas PD. Characterization of immunoreactive forms of human osteocalcin generated in vivo and in vitro. J Bone Miner Res. 1994;9:255-64.

14. Allison J, Stephen H, Richrad E. Measurement of Osteocalcin. Ann Clin Biochem. 2000;37:432-7.

15. Nakashima A, Yorioka N, Mizutani T, Yamagata Z, Ueno T, Takasugi N. Serum cross-linked N-terminal telopeptide of type I collagen for evaluation of renal osteodystrophy in hemodialysis patients. Nephron Clin Pract. 2005;99(3):c78-85.

16. Komaba H, Goto S, Fukagawa M. Critical issues of PTH assays in CKD. Bone. 2009;44:666-70.

17. Kovesdy CP, Ahmadzadeh S, Anderson JE, KalantarZadeh K. Secondary hyperparathyroidism is associated with higher mortality in men with moderate to severe chronic kidney disease. Kidney Int. 2008;73: 1296-302.

18. Ersoy FF, Passadakis SP, Tam P, Memmos ED, Katopodis PK, Ozener C, Akçiçek F, Camsari T, Ateş $\mathrm{K}$, Ataman R, Vlachojannis JG, Dombros AN, Utaş C, Akpolat T, Bozfakioğlu S, Wu G, Karayaylali I, Arinsoy T, Stathakis PC, Yavuz M, Tsakiris JD, Dimitriades CA, Yilmaz ME, Gültekin M, Karayalçin B, Yardimsever M, Oreopoulos DG. Bone mineral density and its correlation with clinical and laboratory factors in chronic peritoneal dialysis patients. J Bone Miner Metab. 2006;24(1):7986.

19. Castillo RF, de la Rosa RJE. Relation between body mass index and bone mineral density among hemodialysis patients with chronic kidney disease. J Ren Care. 2009 Mar;35 Suppl 1:57-64.

20. Piepsz A, Denis R, Ham HR, Dobbeleir A, Schulman C, Erbsmann F. A simple method for measuring separate glomerular filtration rate using a single injection of 99mTc-DTPA and the scintillation camera. J pediatr. 1978;93(5):769-74.

21. Burtis CA, Ashwood AR. Alkaline phosphatase levels in children and adults. In: Burtis CA, Ashwood ER, editors. Tietz fundamentals of clinical chemistry. 5th ed. Philadelphia: WB Saunders; 2001. p. 31-43. 
22. Waller SC, Ridout D, Cantor T, Rees L. Parathyroid hormone and growth in children with chronic renal failure. Kideny Int. 2005;67(23):38-45.

23. Bakr AM. Bone mineral density and bone turnover markers in children with chronic renal failure. Pediatr Nephrol. 2004;19:1390-93.

24. National Kidney Foundation. K/DOQI clinical practice guidelines for bone metabolism and disease in children with chronic kidney disease. Am J Kidney Dis. 2005;46(1):1-121.

25. Malluche HH, Monier-Faugere MC. Renal osteodystrophy: What's in a name? Presentation of a clinically useful new model to interpret bone histologic findings. Clin Nephrol. 2006;65:235-42.

26. Coen G, Ballanti P, Bonucci E, Calabria S, Costantini S, Ferrannini M, Giustini M, Giordano R, Nicolai G, Manni M, Sardella D, Taggi F. Renal osteodystrophy in predialysis and hemodialysis patients: comparison of histologic patterns and diagnostic predictivity of intact PTH. Nephron. 2002;91(1):103-11.

27. Levin A, Bakris GL, Molitch M, Smulders M, Tian J, Williams LA, Andress DL. Prevalence of abnormal serum vitamin D, PTH, calcium, and phosphorus in patients with chronic kidney disease: results of the study to evaluate early kidney disease. Kidney Int. 2007 Jan;71(1):31-8.

28. Wesseling K, Bakkaloglu S, Salusky I. Chronic kidney disease mineral and bone disorder in children. Pediatric Nephrology. 2008;23(2):195-207.

29. Ahmadzadeh A, Ehsan Valavi, Zangeneh-Kamali M, Ahmadzadeh A. Chronic Kidney Disease in Southwestern Iranian Children. Iran J Pediatr. 2009;19(2):147-53.

30. Nilsson P, Melsen F, Malmaeus J, Danielson BG, Mosekilde L. Relationships between calcium and phosphorus homeostasis, parathyroid hormone levels, bone aluminum, and bone histomorphometry in Patients on maintenance hemodialysis. Bone. 1985;6(1):21-7.

31. Vasikaran SD. Utility of biochemical markers of bone turnover and bone mineral density in management of osteoporosis. Crit Rev Clin Lab Sci. 2008;45(2):221-58.

32. Magnusson P, Sharp CA, Magnusson M, Risteli J, Davie MW, Larsson L. Effect of chronic renal failure on bone turnover and bone alkaline phosphatase isoforms. Kidney Int. 2001 Jul;60(1):257-65.

33. Atsumi K, Kushida K, Yamazaki K, Shimizu S, Ohmura A, Inoue T. Risk factors for vertebral fractures in renal osteodystrophy. Am J Kidney Dis. 1999 Feb;33(2):287-93.

34. Groothoff JW, Offringa M, Van Eck-Smit BL, Gruppen MP, Van De Kar NJ, Wolff ED, Lilien MR, Davin JC, Heymans HS, Dekker FW. Severe bone disease and low mineral density after juvenile renal failure. Kidney Int. 2003 Jan;63(1):266-75.

35. Pluskiewicz W, Adamczyk P, Drozdzowska B, Szprynger K, Szczepańska M, Halaba Z, Karasek D. Skeletal status in children and adolescents with chronic renal failure before onset of dialysis or on dialysis. Osteoporos Int. 2003 Jun;14(4):283-88.

36. Ureña P, Bernard-Poenaru O, Ostertag A, Baudoin C, Cohen-Solal M, Cantor T, de Vernejoul MC. Bone mineral density, biochemical markers and skeletal fractures in haemodialysis patients. Nephrol Dial Transplant. 2003 Nov; 18(11):2325-31.

37. Boot AM, Nauta J, de Jong MC, Groothoff JW, Lilien MR, van Wijk JA, Kist-van Holthe JE, Hokken-Koelega AC, Pols HA, de Muinck Keizer-Schrama SM. Bone mineral density, bone metabolism and body composition of children with chronic renal failure with and without growth hormone treatment. Clin Endocrinol (Oxf). 1998 Nov;49(5):665-672. 